\title{
Epidemiology of pyridoxine dependent and pyridoxine responsive seizures in the UK
}

\author{
Peter Baxter
}

\begin{abstract}
Objective-To study the epidemiology of pyridoxine dependent seizures and other forms of pyridoxine responsive seizures. Design-Monthly notifications to the British Paediatric Surveillance Unit over two years. Questionnaire follow up. Setting-UK and the Republic of Ireland. Patients-Children aged 15 years or younger whose seizures respond to pyridoxine.

Interventions-None.

Main outcome measures-Numbers of children with definite, probable, and possible pyridoxine dependent seizures or other seizures responsive to pyridoxine. Results-Point prevalence and birth incidence: $1 / 687000$ and $1 / 783000$, respectively (definite and probable cases); $1 / 317000$ and $1 / 157000$, respectively (all types of pyridoxine responsiveness). Notifications-Pyridoxine dependency: 14 definite, 9 probable, and 10 possible cases; neonatal seizures not meeting case definitions: 7; infantile spasms: 5 . Eight of 18 families of definite/probable cases had 2 affected siblings. Just over a third had atypical presentations and just under a third had features and/or initial diagnoses of birth asphyxia and neonatal hypoxic ischaemic encephalopathy.

Conclusions-Pyridoxine dependency is rare. Atypical presentations are relatively frequent. A trial of pyridoxine is justified in all cases of early onset intractable seizures or status epilepticus, whatever the suspected cause.

(Arch Dis Child 1999;81:431-433)
\end{abstract}

Keywords: pyridoxine dependent seizures; pyridoxine responsive seizures; epidemiology

Early onset seizures responsive to pyridoxine are well known but have been studied only rarely. ${ }^{1}$ A regional population based study found an unexpectedly high prevalence and several previously unreported features, including a specific deficit in expressive speech and an apparent correlation between dose and IQ. ${ }^{2}$ The study was therefore extended to the rest of the UK, through the British Paediatric Surveillance Unit (BPSU). This unit is a part of the Royal College of Paediatrics and Child Health, which studies the epidemiology of rare conditions, usually on a national basis. Pyridoxine dependent seizures were included in their programme with the aims of determining the prevalence of definite or possible pyridoxine dependent seizures in children less than 16 years of age, prospectively studying the incidence in children under 5 years of age, and defining the clinical presentation, natural history, and clinical management of the condition. Here, we report the epidemiological findings.

\section{Methods}

From September 1995 to October 1997, all paediatricians in the UK and the Republic of Ireland were requested to notify children with pyridoxine dependent seizures seen by them in the previous month on a monthly card sent out by the BPSU. Respondents were asked to fill out a questionnaire. Definite cases were defined as neonates, infants, or young children with recurrent (two or more) seizures of any type that cease within seven days of the administration of oral pyridoxine (usual dose, $30 \mathrm{mg}$ / $\mathrm{kg} /$ day; minimum dose, $15 \mathrm{mg} / \mathrm{kg} /$ day; maximum dose, $1000 \mathrm{mg} / \mathrm{kg} /$ day) or within 30 minutes of intravenous pyridoxine (usual dose, $100 \mathrm{mg}$; minimum dose, $50 \mathrm{mg}$ ), that recur when pyridoxine supplementation is withdrawn, and that cease again when pyridoxine is given as above. Possible cases were defined as above, but without an attempt to withdraw pyridoxine. Recurrence of seizures while receiving pyridoxine treatment was an exclusion criterion, unless the recurrence occurred during a febrile illness.

During the study, two subgroups were defined as having "probable" pyridoxine dependency because no formal trial of withdrawal was undertaken. In one subgroup, seizures stopped after a single dose of pyridoxine, recurred later, and again responded to pyridoxine, which was then continued: one child had a formal trial of withdrawal after October 1997 with a positive result and was reclassified as definite. A second subgroup comprised children with an affected sibling whose seizures began at the same age and responded to pyridoxine.

Colleagues were also asked to include patients with pyridoxine responsive seizures in whom there were other suspected or definite causes of seizures, to ensure complete reporting. Children born during the study period and notified after October 1997 have been included, as well as cases notified from other sources. Point prevalence was measured as living children under 16 in October 1997. Denominators were projections from the 1991 population census supplied by the Office for National Statistics for England and Wales, the General Register Office for Scotland, and the Irish Census Office.

Our study was approved by the local ethics committee. 
Table 1 Notifications (all ages) to the BPSU, made directly and seen personally

\begin{tabular}{llllllll}
\hline Origin & Definite & Probable & Possible & IS & Not & Duplicate & Other \\
\hline BPSU & 4 & 7 & 5 & 5 & 13 & 4 & 9 \\
Direct & 3 & 2 & 4 & & 2 & 2 & 2 \\
Personal & 7 & & 1 & 1 & & &
\end{tabular}

"Not" includes the two patients whose questionnaires were not returned; "Other" refers to notifications to the BPSU that were revised, made in error, or lost, and two made directly for an adult and for an undiagnosed sibling who died.

BPSU, British Paediatric Surveillance Unit; IS, infantile spasms.

Table 2 Geographical distribution by place of birth

\begin{tabular}{lllllll}
\hline Region & $\begin{array}{l}\text { Population } \\
\text { (million) }\end{array}$ & D/P cases & All cases & PP & $\begin{array}{l}\text { BPSU } \\
\text { cases }\end{array}$ & $\begin{array}{l}\text { BPSU } \\
\text { non-cases }\end{array}$ \\
\hline Anglia and Oxford & 1.111 & 0 & 0 & - & 0 & 0 \\
North Thames & 1.428 & 3 & 4 & 357 & 1 & 1 \\
Northwest & 1.401 & 4 & 6 & 233 & 6 & 2 \\
Northern and Yorkshire & 1.310 & 4 & 5 & 262 & 0 & 1 \\
South and West & 1.289 & 2 & 4 & 322 & 3 & 1 \\
South Thames & 1.354 & 3 & 3 & 451 & 3 & 2 \\
Trent & 1.038 & 2 & 3 & 346 & 1 & 1 \\
West Midlands & 1.119 & 0 & 2 & 559 & 0 & 2 \\
Wales & 0.602 & 0 & 1 & 602 & 1 & 1 \\
Scotland & 0.959 & 2 & 2 & 479 & 1 & 1 \\
Eire & 1.003 & 0 & 0 & - & 0 & 1
\end{tabular}

D/P, definite and probable cases; "all cases", definite, probable, and possible cases; BPSU cases and non-cases, BPSU notifications, with population data for children aged younger than 16 years in mid-1996 (England and Wales; Eire) and 1997 (Scotland).

BPSU, British Paediatric Surveillance Unit; PP, point prevalence for all cases ( $1 / \mathrm{x} 000$ population).

\section{Results}

Forty seven notifications were received by the BPSU. Twenty five more were obtained via other sources: 15 children and one adult notified directly to the author, seven previously described in the northern regional study, ${ }^{2}$ and two presenting as new cases to the author. Two questionnaires were not returned, but telephone inquiries indicated that neither child fulfilled the case definitions. There were six duplicate notifications.

Including all sources and ages, 14 definite, nine probable, and 10 possible cases were notified (table 1). The point prevalence of definite and probable cases in children less than 16 years of age at the end of the study was $1 / 687000$ and the birth incidence was $1 / 783000$. With the inclusion of possible cases these become $1 / 441000$ and $1 / 313000$, respectively. When all forms of pyridoxine responsive seizures are included the prevalence

Table 3 Year of birth of notifications. Cases born in 1998 are shown for information

\begin{tabular}{|c|c|c|c|c|c|c|}
\hline Birth year & Definite & Probable & Possible & Death & Not & IS \\
\hline 1976 & 1 & & & & & \\
\hline 1977 & & & & 1 & & \\
\hline \multicolumn{7}{|l|}{1978} \\
\hline 1979 & 1 & & & 1 & 1 & \\
\hline 1980 & 3 & & & 1 & & \\
\hline \multicolumn{7}{|l|}{1981} \\
\hline 1982 & & 1 & & & & \\
\hline 1983 & & 1 & & & & \\
\hline 1984 & 2 & & & & & \\
\hline \multicolumn{7}{|l|}{1985} \\
\hline 1986 & & 2 & & & & \\
\hline 1987 & & 1 & & & 1 & \\
\hline 1988 & 2 & & & & & \\
\hline 1989 & & & 1 & & 1 & \\
\hline 1990 & 1 & 1 & & & & \\
\hline 1991 & 2 & & 1 & & 1 & 1 \\
\hline 1992 & & & 1 & & & 1 \\
\hline 1993 & 1 & & & & 1 & 1 \\
\hline 1994 & & 1 & & & & 1 \\
\hline 1995 & 1 & & 4 & & 2 & \\
\hline 1996 & & 2 & & & 5 & 2 \\
\hline 1997 & & & 3 & & 1 & \\
\hline 1998 & & & 3 & & & \\
\hline
\end{tabular}

IS, infantile spasms. becomes $1 / 317000$ and the birth incidence $1 / 157000$.

Four children were notified who presented with West syndrome resistant to vigabatrin and/or steroids but fully responsive to pyridoxine. One idiopathic patient has since discontinued pyridoxine for over three years without any recurrence and with normal development. Two of the others had symptomatic causes. A fifth child with neonatal seizures controlled by pyridoxine and normal early development (classified as a possible case) re-presented at 6 months of age with infantile spasms and hypsarrhythmia that responded clinically and electrophysiologically to an increased dose of pyridoxine: no trial of withdrawal has yet been undertaken. Two other children with West syndrome were notified, one partially responsive and one unresponsive to pyridoxine.

Thirteen patients were notified who did not fulfil the case definitions. Five had neonatal seizures that ceased after the administration of pyridoxine but which did not recur on withdrawal. One of these was initially categorised as a possible case. Two more, both of consanguineous parents, had neonatal seizures that responded to pyridoxine but showed persisting neurological abnormalities and later seizure recurrence. Of the remainder, one had a final diagnosis of benign neonatal sleep myoclonus, whereas others had partial or minimal responses and included children with diagnoses such as Ohtahara syndrome.

Of the remaining notifications, one was an adult, one was a sibling who died without a diagnosis, one was revised to non-epileptic tonic posturing, three were made in error, and five were lost because the consultants who had made the notification had moved without leaving any record. The children concerned could not be traced because all notifications are anonymous and they were not notified separately later.

Eight of the 18 families with definite or probable cases, but none of the families of possible cases, had a positive family history, either with two affected children (five) or with a sibling who developed identical symptoms in the neonatal period and died with intractable seizures (three, all born before 1980). The latter had diagnoses of birth asphyxia (two) and adrenal haemorrhage. The boy to girl ratio was $16: 17$ (definite, $7: 7$; probable, $5: 4$, possible, $4: 6$ ). Racial origins were as follows: definite, 12 white, two Indian; probable, seven white, one Indian, one Indian and Pakistani; possible, eight white, one Indian, one Pakistani. The regional distribution of cases by place of birth was relatively even, with two exceptions, although notifications showed more variability (table 2). The distribution by year of birth was also relatively even, with a rise in possible cases during and after the study (table 3).

Four of each of the definite and probable cases, as well as one possible case, had atypical presentations. These included neonatal onset seizures that initially responded to routine anticonvulsants but recurred from 2 to 8 months of age, or late onset seizures up to the age of 9 months. Of the 20 definite and 
probable cases with neonatal onset seizures, two had one minute Apgar scores of four or less, two more were acidotic at birth, and at least three were believed to have an hypoxic ischaemic encephalopathy before the diagnosis of pyridoxine dependency was established. One or more of these was present in six, as well as in two of eight possible cases.

\section{Discussion}

This is the largest reported study of pyridoxine dependent seizures and the first national population based study. It confirms that all forms of pyridoxine responsive seizures are rare. The higher prevalence of $1 / 100000$ in the northern regional study ${ }^{2}$ is unexplained. No other figures are available for comparison. In the world literature approximately 100 cases have been reported, ${ }^{1}$ of which at least half were diagnosed retrospectively in siblings who had died with symptoms similar to the reported case, and fewer than 40 were definite or probable cases by the criteria used here. The 23 reported in our study contribute greatly to this number.

Our epidemiological data are likely to be accurate. The BPSU achieves returns of 9394\% from paediatricians in the UK and Eire: only $1 \%$ of paediatricians never return forms. ${ }^{3}$ Underrecognition is a problem in all rare conditions, and is suggested here by the high proportion of familial cases, although the absence of siblings dying with similar symptoms since 1980 , together with the relatively even distribution by region and date of birth, suggest that this is not a large number. Several cases notified to the study were recognised late, usually after consultation with a paediatric neurologist. Overestimates of pyridoxine dependent seizures could result from the inclusion of "possible" cases, because these might turn out to be pyridoxine responsive rather than dependent (which occurred in one child during the study), or might include self limiting conditions, such as benign neonatal sleep myoclonus or benign neonatal seizures. In the northern regional study, two possible cases were notified: one of these children has continued pyridoxine, but the other was found to have hypoketotic hypoglycaemia (unpublished data). Again this would be unlikely to affect the results greatly.

Atypical presentations were relatively frequent, occurring in just over one third of cases. Several authors have reported a late presentation up to the age of $2 \frac{1 / 2}{2}$ years, presentation with recurrent episodes of status, initial response to other anticonvulsants, and poor initial response to pyridoxine, although not all of these cases would be considered definite by our study's criteria. ${ }^{4-7}$ Our study did not confirm the suggestion that atypical presentations might be more common than the classic neonatal type. ${ }^{5}$

Our study revealed a group of neonates with seizures that are apparently pyridoxine responsive, but in whom pyridoxine could be withdrawn later without ill effects. They all lacked a family history. Otherwise, there were no obvious distinguishing features. The outcome for development appeared to be good. There is no obvious explanation. Low intakes

\section{Key messages}

- Almost a third of neonatal cases of pyridoxine dependency present with apparent birth asphyxia and/or suspected hypoxic-ischaemic encephalopathy

- All children with early onset (younger than 3 years old) intractable seizures or status should receive a trial of pyridoxine, whatever the suspected cause

of pyridoxine have been reported in a large percentage of pregnant and lactating women, which theoretically could lead to a transient deficiency in some infants. ${ }^{8} \mathrm{~A}$ benign seizure disorder that coincidentally ceased with pyridoxine cannot be excluded. Benign neonatal sleep myoclonus is less likely because they did not have clonic movements.

Infantile spasms responsive to pyridoxine, often in very high doses, are well described, particularly in Japanese publications. ${ }^{9}$ Altogether, approximately $15 \%$ of cases respond, both with symptomatic and idiopathic pathologies, but these children have a better outcome in developmental terms that non-responders. ${ }^{9}$ Most of these are not pyridoxine dependent because pyridoxine can be withdrawn later, as in one child in our study. None of the cases reported here had a positive family history.

Because of the rarity of pyridoxine dependency, trials of pyridoxine for seizures are frequently unrewarding. However pyridoxine in adequate dosage should be given to all neonates or children with difficult seizures or recurrent status beginning before the age of 3 years. Because just under one third of cases with a neonatal onset had low Apgar scores, a low initial $\mathrm{pH}$, and/or a subsequent encephalopathy, this should include all neonates with suspected hypoxic-ischaemic encephalopathy.

This study was only possible through the kind cooperation of paediatric colleagues who notified cases and filled in the questionnaires. Dr R Gilbert, R Lynn, Dr A Nicoll, and members of the British Paediatric Surveillance Unit helped at all stages of its planning and execution. Financial support from the Children Nationwide Medical Research Fund and the Northern acknowledged.

1 Haenggeli CA, Girardin E, Paunier L. Pyridoxine dependent seizures, clinical and therapeutic aspects. Eur $\mathcal{F}$ Paediatr 1991;150:452-5.

2 Baxter P, Griffiths P, Kelly T, Gardner-Medwin D. Pyridoxine dependent seizures. Demographic, clinical, radiological and psychometric features. Effect of dose on IQ. Dev Med and psychometric features. Effect
Child Neurol 1996;39:998-1006.

3 British Paediatric Surveillance Unit. 12th Annual Report 1997-1998. London: Royal College of Paediatrics and Child Health, 1998

4 Ekelund H, Gamstorp I, Von Studnitz W. Apparent response of impaired mental development, minor motor epilepsy and ataxia to pyridoxine. Acta Paediatr Scand 1969;58:572-6.

5 Goutieres F, Aicardi J. Atypical presentations of pyridoxine dependent seizures: a treatable cause of intractable epilepsy in infants. Ann Neurol 1985;17:117-20.

6 Bankier A, Turner M, Hopkins IJ. Pyridoxine dependent seizures-a wider clinical spectrum. Arch Dis Child 1983;58:415-18.

7 Bass NE, Wyllie E, Cohen B, Joseph SA. Pyridoxinedependent epilepsy: the need for repeated pyridoxine trials
and the risk of severe electroclinical suppression with intravenous pyridoxine infusion. F Child Neurol 1996;11:422-4.

8 Guilarte TR. Vitamin B6 and cognitive development: recent Guilarte TR. Vitamin B6 and cognitive development: recent
research findings from human and animal studies. Nutr Rev research findings

9 Ohtahara S, Ohtsuka Y, Yamatogi Y, et al. Prenatal etiologies of West syndrome. Epilepsia 1993;34:716-22. 